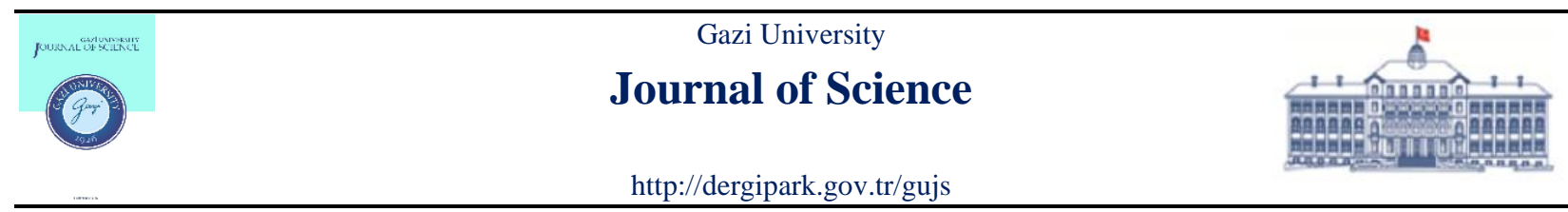

\title{
Experimental Study of the Pressure Effects on Stopping Power for Alpha Particles in Air
}

\author{
Hiwa Mohammad QADR* \\ University of Raparin, College of Science, Department of Physics, Sulaymaneyah, Iraq
}

\author{
Highlights \\ - Measurement of the stopping range and energy loss of $\alpha$-particles in air at various pressure. \\ - In this study, surface barrier silicon detector was used. \\ - Experimental result for stopping power was compared with ICRU and SRIM result.
}

\section{Article Info}

Received: 30 Dec 2020 Accepted: 21 Feb 2021

\section{Keywords}

Stopping power

Mass stopping power

SRIM

Vacuum chamber

Air density

\begin{abstract}
This paper is to investigate stopping power of $\alpha$-particles in air at various pressure of 0 to $1 \mathrm{bar}$, using a surface barrier silicon detector. The energy loss has been obtained at a different distance from 0 to $10 \mathrm{~cm}$. It was found that the energy of the passed $\alpha$-particles has been decreased in a small vacuum chamber with increasing pressure. It is also studied that the amount of $\alpha$-particles reaching the detector follows a decreasing behavior as the distance increases. The result of stopping power was compared with ICRU and SRIM result. Experimental, ICRU and SRIM results for stopping power are closely in good agreement.
\end{abstract}

\section{INTRODUCTION}

In recent years, the general public has become more aware of the sea of radiation that we live in, and its health implications. They are frightened by reports of the high increase in cancer due to exposure to ionizing radiation. Nuclear radiation is said to be ionizing when it possesses a high enough kinetic energy to be able to eject electrons from the atom of any medium it traverses or collides with. This loss of electrons causes the target atom to become positively charged [1,2]. There are two major types of ionizing radiations: directly and indirectly ionizing radiations. The directly ionizing radiations such as the $\alpha$-particle, $\beta$-particles and many other charged particles ionize atoms of their target material due to Columbic collisions with the electrons of the material, and the amount of kinetic energies of these particles determines the amount of this Columbic force $[3,4]$. On the other hand, indirectly ionizing radiations are neutral particles such as high photons and neutrons which do not directly ionize atoms but undergo collisions to eject an energetic electron called the secondary electron [5-10]. Therefore undergoes direct ionization ejecting many other electrons leaving the atom ionized. High energy photons such as the $\mathrm{X}$-rays, $\gamma$-rays and cosmic-rays have the highest penetration power and are therefore the least harmful of the ionizing radiations since the radiation spends little time ionizing the atoms of its target material [11-13]. Whereas, heavy charged particles interact with matter such as $\alpha$-particles which are the least penetrating and hence lose more energy by ionizing atoms of its target [14]. Thus, $\alpha$-particles are the most harmful internal hazard as compared with $\beta$-particles and $\gamma$-rays.

The most important parameters characterizing the energy loss is the stopping power, mass stopping power and range of $\alpha$-particle. Stopping power in different materials has been a subject that has received great in both applied and theoretical science [15,16]. SRIM (The Stopping and Range of Ions in Matter) is a software 
code which calculate the stopping and range of ions into matter using a quantum mechanical treatment of ion-atom collisions [17-20]. Furthermore, Report 49 of the ICRU [21] measured the range and stopping power for different media. In this work, the energy loss of $\alpha$-particles in air at different pressure and distances using silicon Surface barrier detectors is discussed. Then the stopping power value was compared with ICRU and SRIM value.

\section{THEORETICAL CALCULATIONS}

This section summarizes background theoretical relations used for determination of stopping power $S(E)$, Mass stopping power $M S P$, Range $R(E)$. The average energy loss of the $\alpha$-particle $d E$ per unit path length $d x$ is called stopping power [22]. It relies on the energy of the $\alpha$-particles and on the properties of the material it passes which can be written as [23]:

$$
-\frac{d E}{d x}=S(E)
$$

The negative sign makes $S(E)$ positive which is used for the particles lose energy as they pass through the materials [24]. The mass stopping power is obtained by dividing the stopping power by the density (air density $=0.001225 \mathrm{~g} / \mathrm{cm}^{3}$ ) [25]. It refers to the energy lost by the $\alpha$-particles falling on the target as it passes through which is expressed by the following equation [26]:

$$
\frac{S(E)}{\rho}=M S P \text {. }
$$

It is important to consider the range of the $\alpha$-particle in any given medium. The range is defined as the distance of that $\alpha$-particle travel from its source to the target material [27]. This can be calculated using the following relation:

$$
\int_{E_{0}}^{0}\left(\frac{d E}{d x}\right)^{-1} d E=R(E)
$$

Empirically, the average linear range of $\alpha$-particle in air is given as [28]:

$$
\begin{array}{ll}
0.56 E=R_{\text {air }} & \text { for } E<4 \mathrm{MeV} \\
1.24 E-2.62=R_{\text {air }} & \text { for } 4 \leq E<8 \mathrm{MeV} .
\end{array}
$$

In above equation, $E$ is the energy of the particle in $\mathrm{MeV}$.

\section{EXPERIMENTAL PROCEDURE}

The energy loss of $\alpha$-particles throughout air at different distances and different pressures has been investigated in a small vacuum chamber. ANS42 source was used which contains three components of the radioactive source such as ${ }^{241} \mathrm{Am},{ }^{244} \mathrm{Cm}$ and ${ }^{239} \mathrm{Pu}$. These sources emit $\alpha$-radiation with energies of 5485.56, $5804.77,5156.59 \mathrm{keV}$. It was one containing the three isotopes mentioned which are ${ }^{241} \mathrm{Am}$. But, any of the other sources could have been used for finding the optimum voltage of the surface barrier silicon detector. The detector has an area of $450 \mathrm{~mm}^{2}$ and $100 \mu \mathrm{m}$ of depletion depth [29]. Figure 1 shows the schematic view of the experimental setup in which the source and the detector were placed inside a small vacuum chamber. The distance between the source and the detector was $1 \mathrm{~cm}$ for finding the best voltage of the detector. Now the voltage of 50 to $350 \mathrm{~V}$ was applied for $180 \mathrm{~s}$. the corresponding values of channel number and full width at half maximum were recorded to determine the energy resolution. Now, the small vacuum 
chamber was filled with air and the energy of the source inside the small vacuum chamber was measured at different distances from 1 to $10 \mathrm{~cm}$ and for 0 to 1 bar of the air pressure.

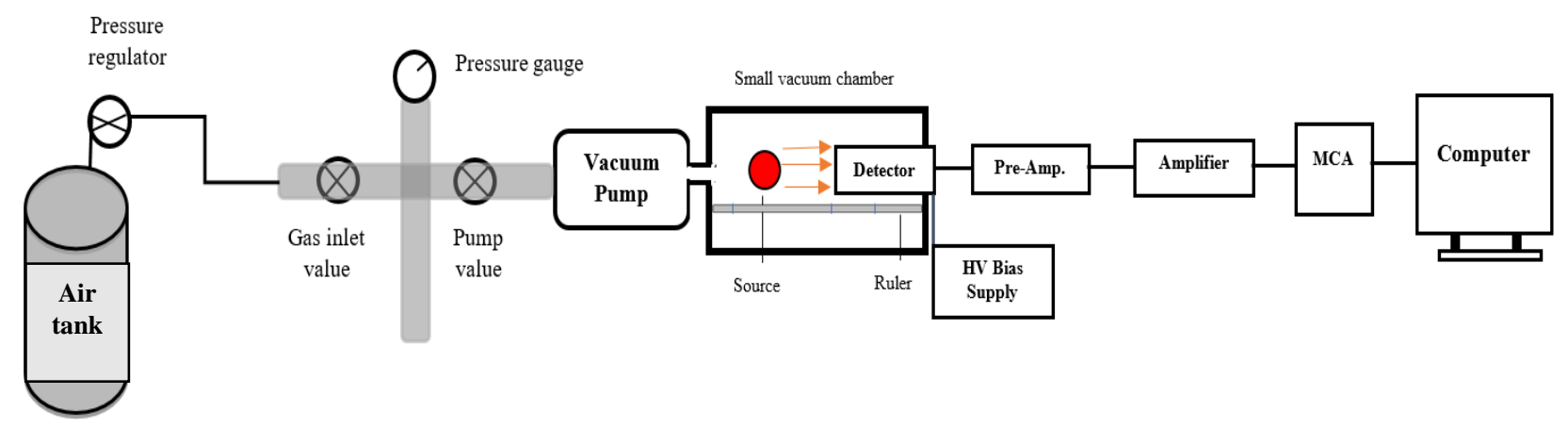

Figure 1. Diagram of experimental setup

\section{RESULTS AND ANALYSIS}

The stopping power of air for $\alpha$-particles at different distances and pressures in the small vacuum chamber was measured. During this study, $\alpha$-particle energy losses in air was determined for ${ }^{241} \mathrm{Am}$ source. The results are shown in Figure 2 that as the pressure in the small vacuum chamber increases, the energy of the $\alpha$-particle decreases. For instant, the measured energy loss of $\alpha$-particles at lower pressure of 0 bar is less since there is almost no molecule of air to interact within the small vacuum chamber. But, the measured energy loss of $\alpha$-particles at a higher pressure of 10 bar is more since the number of air molecules in the traversing path of the $\alpha$-particle is larger, which results in more collisions. Nevertheless, the energy loss of $\alpha$-particle increases as distance from the source to detector increases in the vacuum chamber. More precisely, Figure 2 shows that at a pressure equal to 1 bar, the energy of the $\alpha$-particle is lost completely at distances equal from 5.8 to $10 \mathrm{~cm}$. 

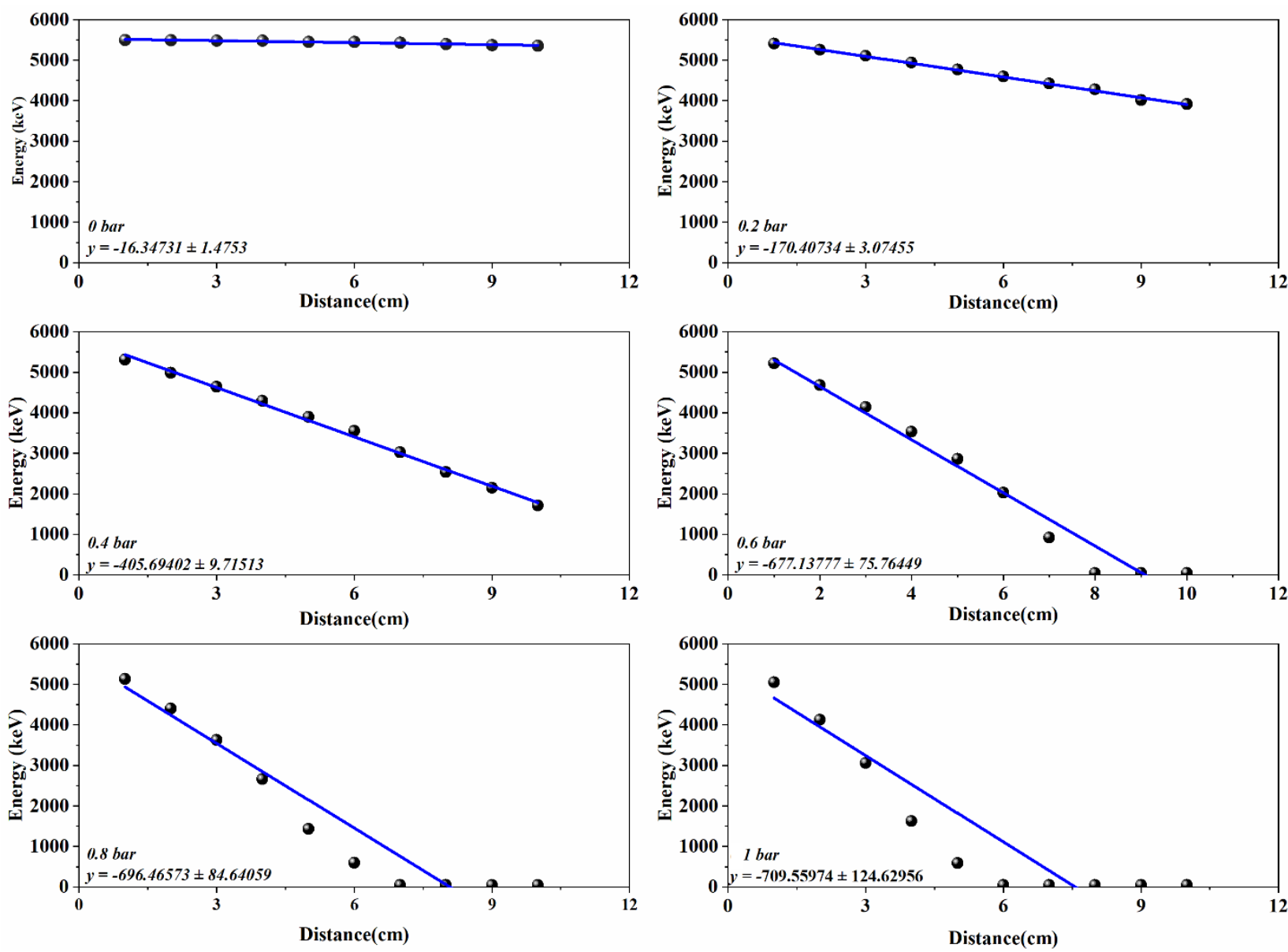

Figure 2. Energy loss of alpha particles in air as a function of distance for different values of pressure

The overall calculated results of the stopping power of $\alpha$-particles in air have been shown in Figure 3. In this figure, the stopping power is low at lower pressure of 0 bar whereas the stopping power increases when the pressure is increasing. The values of mass stopping power of $\alpha$-particles in air at different pressure have been presented in Table 1. They have been calculated by Equation (2). The results show that the mass stopping power increases as the air pressure increases.

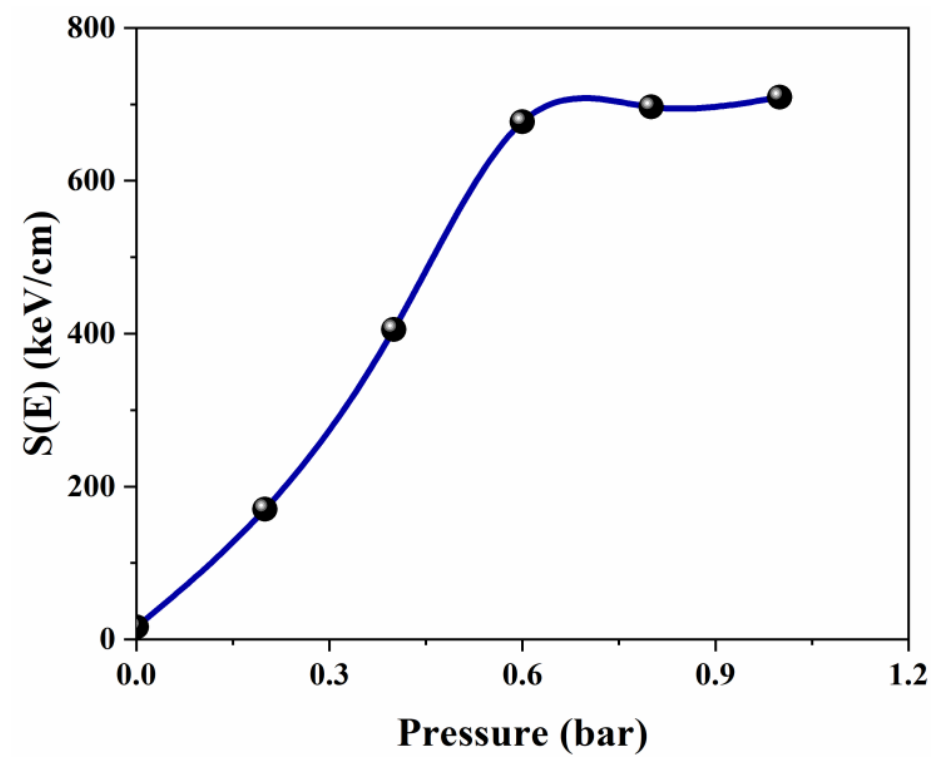

Figure 3. Stopping power as a function of air pressure 
Table 1. The mass stopping power of $\alpha$-particle in air at different values of pressure

\begin{tabular}{|c|c|}
\hline Pressure (bar) & $M S P\left(\mathrm{keV} \mathrm{cm}^{2} / \mathrm{g}\right)$ \\
\hline 0 & 13344.74 \\
\hline 0.2 & 139108.03 \\
\hline 0.4 & 331178.8 \\
\hline 0.6 & 552765.5 \\
\hline 0.8 & 568543.45 \\
\hline 1.0 & 579232.41 \\
\hline
\end{tabular}

Comparison of the $\alpha$-particle energy losses in air obtained from data of ICRU [21] and SRIM code [30]. They provide the stopping power values of $\alpha$-particles in the energy range of 100-5000 keV. Compared to the results of both calculations in Figure 4 that as the energy increases from 100 to $800 \mathrm{keV}$, the stopping power increases too until a minimum is reached, after that the behavior of the stopping power is exactly difference, it decreases dramatically until the end of the figure. The predicted values based on the ICRU show good agreement with the SRIM code.

Table 2 shows the result of the value of the measured range and stopping power for air. An independent check of the discrepancies among the experimental, ICRU and SRIM value can be performed by looking at the inferred $\alpha$-particle ranges in air. A commonly used empirical formula from Equation (5) that the range with the energy of $5.4856 \mathrm{keV}$ to be $4.18 \mathrm{~cm}$, which is very close to the experimental and ICRU and SRIM value. Furthermore, the range for experimental value is $5.8 \mathrm{~cm}$ at pressure $1 \mathrm{bar}$, therefore the energy became zero at a distance of $5.8 \mathrm{~cm}$ as shown in Figure 2. Notice that in Table 2, the larger range has the shorter stopping power, thus the stopping power for experimental value is shorter than SRIM and ICRU.

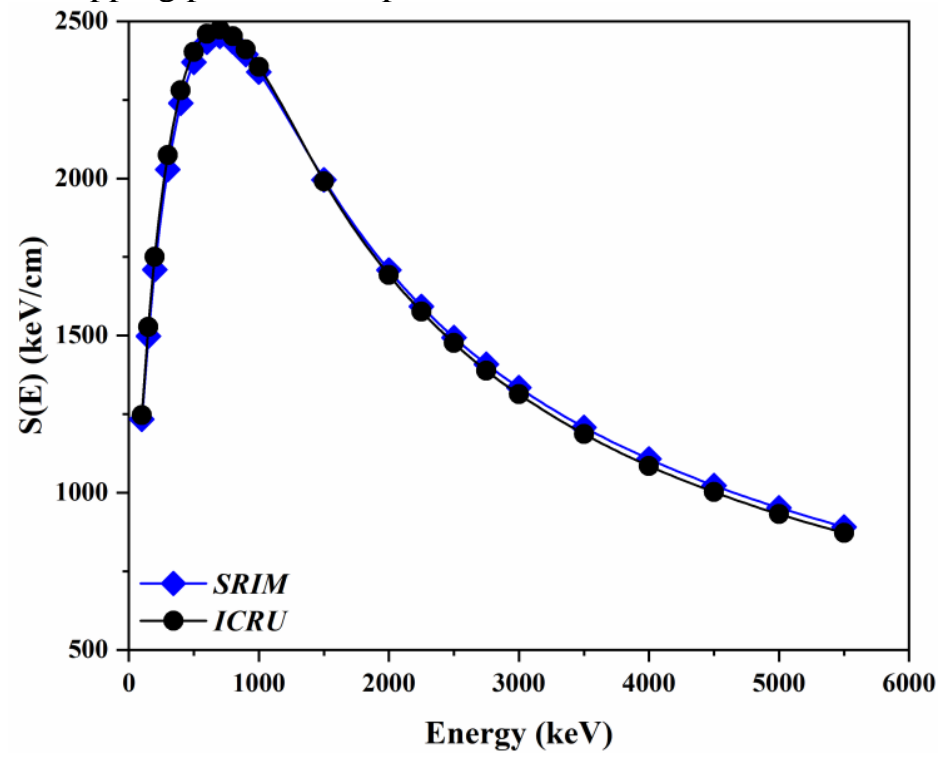

Figure 4. Stopping power as a function of ion energy for SRIM and ICRU

Table 2. The value of the experimental, SRIM and ICRU value for range and stopping power in air

\begin{tabular}{|c|c|c|c|c|c|c|}
\hline \multirow{3}{*}{ Air } & \multicolumn{3}{|c|}{ Range (cm) } & \multicolumn{3}{c|}{ Stopping power (keV/cm) } \\
\cline { 2 - 7 } & Experimental & SRIM & ICRU & Experimental & SRIM & ICRU \\
\cline { 2 - 7 } & 5.8 & 4.1 & 4.76 & 709.5597 & 882.245 & 863.168 \\
\hline
\end{tabular}




\section{CONCLUSIONS}

The stopping power values of air for ${ }^{241} \mathrm{Am}$ source with pressures 0 to 1 bar have been investigated. It was observed that as the pressure is increased, the energy loss of $\alpha$-particle decreases due to the traversing path of the alpha particle in air when the distance between the detector and source decreases. As the pressure increases to 1 bar, the energy loss of alpha particles increases more due to the increase in the amount of interaction with the molecules. The results of the stopping power and range were compared with SRIM and ICRU, showing a good agreement with experimental results.

\section{CONFLICTS OF INTEREST}

No conflict of interest was declared by the author.

\section{REFERENCES}

[1] Charlesby, A., Atomic Radiation and Polymers: International Series of Monographs on Radiation Effects in Materials, 1st Edition, Elsevier, (2016).

[2] Schippers, S., Sokell, E., Aumayr, F., Sadeghpour, H., Ueda, K., Bray, I., Bartschat, K., Murray, A., Tennyson, J., Dorn, A., "Roadmap on Photonic, Electronic and Atomic Collision Physics: Ii. Electron and Antimatter Interactions", Journal of Physics B: Atomic, Molecular and Optical Physics, 52(17): 171002, (2019).

[3] Çalişkan, B., Çalişkan, A. C., "Interaction with Matter of Ionizing Radiation and Radiation Damages (Radicals)", Ionizing Radiation Effects and Applications: 135, (2018).

[4] Gandini, M., Villa, I., Beretta, M., Gotti, C., Imran, M., Carulli, F., Fantuzzi, E., Sassi, M., Zaffalon, M., Brofferio, C., "Efficient, Fast and Reabsorption-Free Perovskite Nanocrystal-Based Sensitized Plastic Scintillators", Nature Nanotechnology, 3: 1-7, (2020).

[5] Qadr, H. M., "Comparison of Energy Resolution and Efficiency of Nai (Ti) and Hpge Detector Using Gamma-Ray Spectroscopy", Journal of Physical Chemistry and Functional Materials, 3(1): 24-7, (2020).

[6] Hamad, A. M., Qadr, H. M., "Gamma-Rays Spectroscopy by Using a Thallium Activated Sodium Iodide Nai (Ti)", Eurasian Journal of Science and Engineering, 4(1): 99-111, (2018).

[7] Alcocer, G., Alcocer, P., Marquez, C., "Burns by Ionizing and Non-Ionizing Radiation", Journal of Burn Care \& Research, (2020).

[8] Qadr, H. M., "Proportional Counter in X-Ray Fluorescence", Aksaray University Journal of Science and Engineering, 5(1): 1-7, (2021).

[9] Doğan, M., Meriç, N., KadioğLu, Y., Samet, R., "Gis Approach to Radioactive Contamination around Seyitömer Thermic Powerhouse", Gazi University Journal of Science, 23(2): 137-48, (2010).

[10] Haciyakupoğlu, S., Tezsezer, S., Orucoglu, E., "Determination of 40k in Beach Sand and Seawater Samples at Sarımsaklı Beach of Aegean Sea (Turkey)", Gazi University Journal of Science, 24(3): 495500, (2011).

[11] Qadr, H. M., "Calculation for Gamma Ray Buildup Factor for Aluminium, Graphite and Lead", International Journal of Nuclear Energy Science and Technology, 13(1): 61-9, (2019). 
[12] Qadr, H. M., "Calculation of Gamma-Ray Attenuation Parameters for Aluminium, Iron, Zirconium and Tungsten", Problems of Atomic Science and Technology, Ser Thermonuclear Fusion, 43(2): $25-$ $30,(2020)$.

[13] Phillips, G., Monaghan, W. P., "Radiation Safety for Anesthesia Providers", AANA Journal, 79(3), (2011).

[14] Weaver, B. A., Westphal, A. J., "Energy Loss of Relativistic Heavy Ions in Matter", Nuclear Instruments and Methods in Physics Research Section B: Beam Interactions with Materials and Atoms, 187(3): 285-301, (2002).

[15] Yang, M., Virshup, G., Clayton, J., Zhu, X. R., Mohan, R., Dong, L., "Theoretical Variance Analysis of Single-and Dual-Energy Computed Tomography Methods for Calculating Proton Stopping Power Ratios of Biological Tissues", Physics in Medicine \& Biology, 55(5): 1343, (2010).

[16] Northcliffe, L. C., Schilling, R. F., "Range and Stopping-Power Tables for Heavy Ions", Atomic Data and Nuclear Data Tables, 7(3-4): 233-463, (1970).

[17] Qadr, H. M., Hamad, A. M., "Using of Stopping and Range of Ions in Matter Code to Study of Radiation Damage in Materials", RENSIT, 12(4): 451-6, (2020).

[18] Qadr, H. M., "Effect of Ion Irradiation on the Mechanical Properties of High and Low Copper", Atom Indonesia, 46(1): 47-51, (2020).

[19] Qadr, H. M., "A Molecular Dynamics Calculation to Cascade Damage Processes", The Annals of "Dunarea de Jos", University of Galati Fascicle IX, Metallurgy and Materials Science, 43(4): 13-6, (2020).

[20] Qadr, H. M., "Effect of Ion Irradiation on the Hardness Properties of Zirconium Alloy", Annals of the University of Craiova, Physics, 29: 68-76, (2019).

[21] ICRU., "Stopping Powers and Ranges for Protons and Alpha Particles", ICRU Bethesda, USA, (1993).

[22] Ahmed, I., Nowrin, H., Dhar, H., "Stopping Power and Range Calculations of Protons in Human Tissues", Baghdad Science Journal, 17(4), (2020).

[23] Saied, B. M., Younis, T. A., Shbeeb, A. K., "Mass Stopping Power of Alpha Particles in Liquid Water and Some Gases", AIP Conference Proceedings, (2019).

[24] Kavaz, E., Tekin, H., Agar, O., Altunsoy, E., Kilicoglu, O., Kamislioglu, M., Abuzaid, M., Sayyed, M. J. C. I., "The Mass Stopping Power/Projected Range and Nuclear Shielding Behaviors of Barium Bismuth Borate Glasses and Influence of Cerium Oxide", 45(12): 15348-57, (2019).

[25] Sy, N. T., Beer, S., Konečný, P., Van Bien, V., "Simulation Study of Shaped Charge Collapse Process and Optimal Standoff Determination", 2019 International Conference on Military Technologies (ICMT), (2019).

[26] Perişanoğlu, U., "Assessment of Nuclear Shielding and Alpha/Proton Mass Stopping Power Properties of Various Metallic Glasses", Applied Physics A, 125(11): 801, (2019).

[27] Hiwa, M. Q., "Stopping Power of Alpha Particles in Helium Gas", Bulletin of the Moscow State Technical University NE Bauman Series "Natural Sciences", (2): 117-25, (2020). 
[28] Yu, K. N., Yip, C. W. Y., Nikezic, D., Ho, J. P. Y., Koo, V. S. Y., "Comparison among Alpha-Particle Energy Losses in Air Obtained from Data of Srim, Icru and Experiments", Applied Radiation and Isotopes, 59(5-6): 363-6, (2003).

[29] Carvalho, F., Oliveira, J., "Alpha Emitters from Uranium Mining in the Environment", Journal of Radioanalytical and Nuclear Chemistry, 274(1): 167-74, (2007).

[30] Ziegler, J. F., Biersack, J. P., Ziegler, M. D., "The Stopping and Range of Ions in Matter", SRIM: http://www.srim.org, (2009). 